\title{
Effect of Different Manurial Practices on Nutrient Availability at Different Growth Stages under SRI Method of Rice Planting
}

\author{
Sowmya Pogula* and K.K. Rout \\ Department of Soil Science \& Agricultural Chemistry, Orissa University of Agricultural \\ Chemistry, Bhubaneswar 751003, India \\ *Corresponding author
}

\section{A B S T R A C T}

\section{Keywords}

Active tillering,

Panicle initiation,

Biofertilizers,

Microbial activity

Article Info

Accepted:

20 April 2018

Available Online:

10 May 2018
The field experiment was conducted during kharif and rabi season of 2106 to study the effect of different manurial practices on soil microbial activity under SRI method of rice planting. The field experiment was laid out in Randomized block design with 8 treatments replicated thrice. The treatments include incorporation of $100 \% \mathrm{RD}\left(80: 40: 40 \mathrm{~N}: \mathrm{P}_{2} \mathrm{O}_{5}: \mathrm{K}_{2} \mathrm{O}\right.$ $\mathrm{Kg} / \mathrm{ha}$ ) with $10 \mathrm{tFYM} / \mathrm{ha}$ in $\mathrm{T} 1,100 \% \mathrm{RD}$ with $5 \mathrm{tFYM} /$ ha, green manure and biofertilizer in T2, 75\% RDF with Nimin + FYM 10t /ha in T3, 75\% RDF with Nimin + FYM 5t/ha + Green Manuring + Biofertilizer inT4, STBR (soil test based fertilizer recommendation) with 10t FYM/ha in T5, STBR with 5tFYM/ha, green manure and biofertilizer in T6, $100 \% \mathrm{RD}$ in $\mathrm{T} 7$ and $100 \%$ organic in $\mathrm{T} 8$. The results revealed that treatment treated with STBR with 5tFYM/ha, green manure and biofertilizer showed best result and highest found during active tillering in nutrient availability in all treatments and also showed increase in microbial population and enzyme activity during panicle initiation in all the treatments.

\section{Introduction}

Farmers in a number of countries have been able to increase the yields from their current rice varieties with available resources by utilizing what is known as the system of rice intensification (SRI) (Kabir and Uphoff, 2007; Namara et al., 2008; Sato and Uphoff, 2007; Sinha and Talati, 2007). Higher productivity is achieved by making certain changes in the management of rice plants and the resources upon which these depend-soil nutrients, air, water, soil biota, and solar energy (Ceesay et al., 2007; Lin et al., 2009; Thakur et al., 2010; Zhao et al., 2009).
The changes in practice that constitute this alternative cultural system for growing rice are reviewed in the "Discussion' section. There we consider how SRI management could affect two principal factors that apparently contribute to the productivity gains observed: enhanced size and functioning of plant root systems, and more abundant and diverse soil biota (Mishra and Salokhe, 2008; Thakur et al., 2010; Zhao et al., 2010). That SRI agronomic concepts and practices are being extended successfully now also to rainfed rice cultivation and to other crops besides rice (http://sri.ciifad.cornell.edu/aboutsri/othercrop s/ index.html) suggests that there are positive 
impacts on the functioning of soil systems and/or on plant-soil interactions rather than just on the crop plants themselves.

Several studies have documented the effects of SRI management practices on root development and functioning, e.g., Mishra and Salokhe (2008) and Thakur et al., (2010). Fewer have focused on the effects of these practices on the soil biota, exceptions being Sooksa-Nguan et al., (2009) and Zhao et al., (2010). In this issue of PAWE, Mishra and Salokhe (2011) and Lin et al., (2011) present some further evidence on this subject.

\section{Materials and Methods}

SRI cultivation rice plants are transplanted singly and with wide spacing in a square pattern, radically reducing plant population. This practice which differs from usual practice which assume more plants will give more yield, gives the plants root system more room to grow (Thakur et al., 2010). Soil fertility being enhanced by the combination of plantsoil- water- nutrient- microbial interactions supported by SRI practices, making otherwise unavailable nutrient available through microbiological process (Uphoffs, 2003). Hence it is ideally suitable for resource poor farmers.

The field experiment was conducted during kharif and rabi season of 2106 to study the effect of different manurial practices on rice yield and soil biological properties under SRI method of rice planting. The field experiment was laid out in Randomized block design with 8 treatments replicated thrice. The treatments include incorporation of $100 \% \mathrm{RD}(80: 40: 40$ $\mathrm{N}: \mathrm{P} 2 \mathrm{O} 5: \mathrm{K} 2 \mathrm{O} \mathrm{Kg} / \mathrm{ha}$ ) with $10 \mathrm{t} \mathrm{FYM} / \mathrm{ha}$ in $\mathrm{T} 1$, $100 \% \mathrm{RD}$ with $5 \mathrm{tFYM} / \mathrm{ha}$, green manure and biofertilizer in $\mathrm{T} 2,75 \% \mathrm{RDF}$ with Nimin + FYM 10t /ha in T3, 75\% RDF with Nimin + FYM 5t/ha + Green Manuring + Biofertilizer in T4, STBR (soil test based fertilizer recommendation) with $10 \mathrm{tFYM} / \mathrm{ha}$ in $\mathrm{T} 5$,
STBR with 5tFYM/ha, green manure and biofertilizer in T6, 100\% RD in T7 and 100\% organic in $\mathrm{T} 8$.

\section{Results and Discussion}

The effect of different manures on nitrogen availability at different growth stages showed that the nitrogen availability was highest in the treatement six treated with STBR+FYM 5t /ha + Green Manuring + Biofertilizer and found highest in the active tillering stages and gradually decreases up to maturity this due to the increase in the uptake of nitrogen. The effect of different manures on phosphorous availability at different growth stages showed that the nitrogen availability was highest in the treatment six treated with STBR+FYM 5t /ha + Green Manuring + Biofertilizer and found highest in the active tillering stages and gradually decreases up to maturity this due to the increase in the uptake of phosphorous (Table 1 and 2).

The effect of different manures on potassium availability at different growth stages showed that the nitrogen availability was highest in the treatment six treated with STBR+FYM 5t /ha + Green Manuring + Biofertilizer and found highest in the active tillering stages and gradually decreases up to maturity this due to the increase in the uptake of phosphorous (Table 3).

Concurrent studies of Gyathry (2002) investigated such effects directly. Her assessments of the impact of SRI management practices documented how changes in cultural techniques could alter the microbial profile as well as the abundance of beneficial soil microorganisms. The SRI practices assessed included: younger seedlings, soil-aerating weeding with a mechanical weeder, water management to avoid continuous soil saturation, and green manures to enhance soil organic matter. These practices, in combination, had positive effects on soil biota. 
Table.1 Effect of different manures on nitrogen availability $(\mathrm{kg} / \mathrm{ha})$ at different growth stages

\begin{tabular}{|c|c|c|c|c|c|}
\hline $\begin{array}{l}\text { Sl. } \\
\text { No. }\end{array}$ & Treatments & $\begin{array}{l}\text { Active } \\
\text { tillering }\end{array}$ & $\begin{array}{l}\text { Panicle } \\
\text { initiation }\end{array}$ & Flowering & Maturity \\
\hline $\mathbf{T}_{1}$ & $100 \%$ RDF + FYM 10t /ha & 188 & 176.12 & 164.21 & 152.17 \\
\hline $\mathbf{T} 2$ & $\begin{array}{l}\text { 100\% RDF + FYM 5t/ha } \\
\text { +Green Manuring + } \\
\text { Biofertilizer }\end{array}$ & 176 & 162.1 & 153.21 & 148.19 \\
\hline$\overline{T_{3}}$ & $\begin{array}{l}75 \% \text { RDF with Nimin }+ \\
\text { FYM 10t /ha }\end{array}$ & 184 & 174.34 & 163.11 & 154.32 \\
\hline $\mathbf{T}_{4}$ & $\begin{array}{l}\text { 75\% RDF with Nimin + } \\
\text { FYM 5t/ha + Green } \\
\text { Manuring + Biofertilizer }\end{array}$ & 167 & 153.71 & 146.27 & 136.21 \\
\hline $\mathbf{T}_{5}$ & STBR + FYM 10t /ha & 154.18 & 144.1 & 134.21 & 122.24 \\
\hline $\mathbf{T}_{6}$ & $\begin{array}{l}\text { STBR+ FYM 5t /ha + Green } \\
\text { Manuring + Biofertilizer }\end{array}$ & 190 & 187.22 & 175.33 & 164.32 \\
\hline$\overline{T_{7}}$ & $100 \% \mathrm{RDF}$ & 163.15 & 154.22 & 148.32 & 132.11 \\
\hline$\overline{T_{8}}$ & $\begin{array}{l}100 \% \text { Organic (Green } \\
\text { manuring + FYM + Mataka } \\
\text { khata+ Biofertilizer) }\end{array}$ & 154.11 & 143.38 & 137.22 & 126.32 \\
\hline
\end{tabular}

Table.2 Effect of different manures on phosphorous availability (kg/ha) at Different growth stages

\begin{tabular}{|c|c|c|c|c|c|}
\hline $\begin{array}{l}\text { Sl. } \\
\text { No. }\end{array}$ & Treatments & $\begin{array}{l}\text { Active } \\
\text { tillering }\end{array}$ & $\begin{array}{c}\text { Panicle } \\
\text { initiation }\end{array}$ & Flowering & Maturity \\
\hline$T_{1}$ & $100 \%$ RDF + FYM 10t /ha & 21.11 & 18.23 & 17.12 & 15.32 \\
\hline T2 & $\begin{array}{l}100 \% \text { RDF + FYM 5t/ha } \\
\text { +Green Manuring + } \\
\text { Biofertilizer }\end{array}$ & 19.23 & 16.32 & 14.21 & 12.11 \\
\hline$\overline{T_{3}}$ & $\begin{array}{l}75 \% \text { RDF with Nimin }+ \\
\text { FYM 10t /ha }\end{array}$ & 27.15 & 21.11 & 18.17 & 15.51 \\
\hline $\mathbf{T}_{4}$ & $\begin{array}{l}\text { 75\% RDF with Nimin + FYM } \\
\text { 5t/ha + Green Manuring + } \\
\text { Biofertilizer }\end{array}$ & 25.21 & 20.24 & 18.32 & 16.33 \\
\hline$T_{5}$ & STBR + FYM 10t /ha & 18.22 & 14.22 & 13.21 & 11.10 \\
\hline $\mathrm{T}_{6}$ & $\begin{array}{l}\text { STBR+ FYM 5t /ha + Green } \\
\text { Manuring + Biofertilizer }\end{array}$ & 32.14 & 27.32 & 25.54 & 22.11 \\
\hline $\mathbf{T}_{7}$ & $100 \% \mathrm{RDF}$ & 19.32 & 16.20 & 13.24 & 10.11 \\
\hline$\overline{T_{8}}$ & $\begin{array}{l}100 \% \text { Organic (Green } \\
\text { manuring + FYM + Mataka } \\
\text { khata+ Biofertilizer) }\end{array}$ & 22.32 & 15.27 & 11.11 & 9.04 \\
\hline
\end{tabular}


Table.3 Effect of different manures on potassium availability $(\mathrm{kg} / \mathrm{ha})$ at different growth stages

\begin{tabular}{|c|c|c|c|c|c|}
\hline $\begin{array}{l}\text { Sl. } \\
\text { No. }\end{array}$ & Treatments & $\begin{array}{l}\text { Active } \\
\text { tillering }\end{array}$ & $\begin{array}{l}\text { Panicle } \\
\text { initiation }\end{array}$ & Flowering & Maturity \\
\hline $\mathbf{T}_{1}$ & $100 \%$ RDF + FYM 10t /ha & 73.11 & 68.12 & 65.32 & 62.11 \\
\hline $\mathrm{T} 2$ & $\begin{array}{l}\text { 100\% RDF + FYM 5t/ha } \\
\text { +Green Manuring + } \\
\text { Biofertilizer }\end{array}$ & 60.03 & 57.03 & 55.01 & 52.18 \\
\hline $\mathbf{T}_{\mathbf{3}}$ & $\begin{array}{l}75 \% \text { RDF with Nimin }+ \\
\text { FYM 10t /ha }\end{array}$ & 61.44 & 54.22 & 50.12 & 47.02 \\
\hline $\mathbf{T}_{4}$ & $\begin{array}{l}75 \% \text { RDF with Nimin + } \\
\text { FYM 5t/ha + Green } \\
\text { Manuring + Biofertilizer }\end{array}$ & 64.07 & 51.11 & 48.11 & 45.12 \\
\hline $\mathrm{T}_{5}$ & STBR + FYM 10t /ha & 60.12 & 56.21 & 54.10 & 48.11 \\
\hline $\mathrm{T}_{6}$ & $\begin{array}{l}\text { STBR+ FYM 5t /ha + Green } \\
\text { Manuring + Biofertilizer }\end{array}$ & 74.21 & 69.22 & 65.21 & 62.10 \\
\hline$\overline{T_{7}}$ & $100 \% \mathrm{RDF}$ & 61.10 & 54.21 & 52.01 & 51.05 \\
\hline $\mathrm{T}_{8}$ & $\begin{array}{l}100 \% \text { Organic (Green } \\
\text { manuring + FYM + Mataka } \\
\text { khata+ Biofertilizer) }\end{array}$ & 54.21 & 51.11 & 47.21 & 43.21 \\
\hline
\end{tabular}

Table.4 Effect of different manures on bacteria population (log 10 g-1 dry soil) at different growth stages

\begin{tabular}{|c|c|c|c|c|c|}
\hline $\begin{array}{l}\text { S. } \\
\text { No. }\end{array}$ & Treatments & $\begin{array}{l}\text { Active } \\
\text { tillering }\end{array}$ & $\begin{array}{l}\text { Panicle } \\
\text { initiation }\end{array}$ & Flowering & Maturity \\
\hline $\mathbf{T}_{1}$ & $100 \%$ RDF + FYM 10t /ha & 4.1 & 7.12 & 3.28 & 3.01 \\
\hline $\mathrm{T} 2$ & $\begin{array}{l}\text { 100\% RDF + FYM 5t/ha } \\
\text { +Green Manuring + } \\
\text { Biofertilizer }\end{array}$ & 4.34 & 6.20 & 3.35 & 2.87 \\
\hline $\mathbf{T}_{3}$ & $\begin{array}{l}75 \% \text { RDF with Nimin }+ \\
\text { FYM 10t /ha }\end{array}$ & 3.54 & 4.10 & 3.21 & 2.11 \\
\hline $\mathbf{T}_{4}$ & $\begin{array}{l}75 \% \text { RDF with Nimin + } \\
\text { FYM 5t/ha + Green } \\
\text { Manuring + Biofertilizer }\end{array}$ & 3.15 & 4.02 & 2.67 & 1.98 \\
\hline $\mathbf{T}_{5}$ & STBR + FYM 10t /ha & 4.34 & 5.89 & 3.21 & 2.19 \\
\hline $\mathrm{T}_{6}$ & $\begin{array}{l}\text { STBR+ FYM 5t /ha + Green } \\
\text { Manuring + Biofertilizer }\end{array}$ & 5.45 & 9.22 & 3.10 & 2.15 \\
\hline$\overline{T_{7}}$ & $100 \% \mathrm{RDF}$ & 3.54 & 4.10 & 3.01 & 2.09 \\
\hline $\mathbf{T}_{8}$ & $\begin{array}{l}100 \% \text { Organic (Green } \\
\text { manuring + FYM + Mataka } \\
\text { khata+ Biofertilizer) }\end{array}$ & 4.23 & 5.04 & 3.06 & 2.11 \\
\hline
\end{tabular}


Table.5 Effect of different manures on fungi population ( $\log 10 \mathrm{~g}^{-1}$ dry soil) at different growth stages

\begin{tabular}{|c|c|c|c|c|c|}
\hline $\begin{array}{l}\text { Sl. } \\
\text { No. }\end{array}$ & Treatments & $\begin{array}{l}\text { Active } \\
\text { tillering }\end{array}$ & $\begin{array}{l}\text { Panicle } \\
\text { initiation }\end{array}$ & Flowering & Maturity \\
\hline $\mathbf{T}_{1}$ & $100 \%$ RDF + FYM 10t /ha & 4.22 & 6.12 & 3.12 & 2.91 \\
\hline$\overline{\mathrm{T} 2}$ & $\begin{array}{l}\text { 100\% RDF + FYM 5t/ha } \\
\text { +Green Manuring + } \\
\text { Biofertilizer }\end{array}$ & 4.53 & 5.22 & 4.01 & 3.23 \\
\hline $\mathbf{T}_{\mathbf{3}}$ & $\begin{array}{l}75 \% \text { RDF with Nimin }+ \\
\text { FYM 10t /ha }\end{array}$ & 4.13 & 5.29 & 4.05 & 3.95 \\
\hline $\mathbf{T}_{4}$ & $\begin{array}{l}\text { 75\% RDF with Nimin + } \\
\text { FYM 5t/ha + Green } \\
\text { Manuring + Biofertilizer }\end{array}$ & 4.22 & 5.11 & 3.97 & 3.27 \\
\hline $\mathbf{T}_{5}$ & STBR + FYM 10t /ha & 4.22 & 4.02 & 3.47 & 2.19 \\
\hline $\mathbf{T}_{6}$ & $\begin{array}{l}\text { STBR+ FYM 5t /ha + Green } \\
\text { Manuring + Biofertilizer }\end{array}$ & 6.56 & 8.22 & 5.12 & 4.82 \\
\hline$\overline{T_{7}}$ & $100 \% \mathrm{RDF}$ & 4.34 & 5.01 & 3.01 & 2.65 \\
\hline $\mathbf{T}_{8}$ & $\begin{array}{l}\text { 100\% Organic (Green } \\
\text { manuring + FYM + Mataka } \\
\text { khata+ Biofertilizer) }\end{array}$ & 5.22 & 4.19 & 3.10 & 2.44 \\
\hline
\end{tabular}

Table.6 Effect of different manures on actinomycetes population ( $\log 10 \mathrm{~g}^{-1}$ dry soil) at different growth stages

\begin{tabular}{|c|c|c|c|c|c|}
\hline $\begin{array}{l}\text { Sl. } \\
\text { No. }\end{array}$ & Treatments & $\begin{array}{l}\text { Active } \\
\text { tillering }\end{array}$ & $\begin{array}{c}\text { Panicle } \\
\text { initiation }\end{array}$ & Flowering & Maturity \\
\hline $\mathrm{T}_{1}$ & $100 \%$ RDF + FYM 10t /ha & 4.53 & 6.32 & 3.21 & 3.03 \\
\hline T2 & $\begin{array}{l}\text { 100\% RDF + FYM 5t/ha } \\
\text { +Green Manuring + } \\
\text { Biofertilizer }\end{array}$ & 4.21 & 5.26 & 3.54 & 3.23 \\
\hline$\overline{T_{3}}$ & $\begin{array}{l}\text { 75\% RDF with Nimin + } \\
\text { FYM 10t /ha }\end{array}$ & 3.55 & 4.65 & 3.21 & 2.78 \\
\hline$\overline{T_{4}}$ & $\begin{array}{l}\text { 75\% RDF with Nimin + } \\
\text { FYM 5t/ha + Green } \\
\text { Manuring + Biofertilizer }\end{array}$ & 3.56 & 4.12 & 3.01 & 2.67 \\
\hline$T_{5}$ & STBR + FYM 10t /ha & 4.67 & 5.02 & 3.22 & 3.04 \\
\hline $\mathrm{T}_{6}$ & $\begin{array}{l}\text { STBR+ FYM 5t /ha + Green } \\
\text { Manuring + Biofertilizer }\end{array}$ & 5.55 & 7.76 & 4.12 & 3.29 \\
\hline $\mathrm{T}_{7}$ & $100 \% \mathrm{RDF}$ & 3.67 & 4.12 & 3.01 & 2.56 \\
\hline $\mathbf{T}_{8}$ & $\begin{array}{l}\text { 100\% Organic (Green } \\
\text { manuring + FYM + Mataka } \\
\text { khata+ Biofertilizer) }\end{array}$ & 4.33 & 5.03 & 3.95 & 2.44 \\
\hline
\end{tabular}


Table.7 Effect of different manures on dehydrogenase activity ( $\mu$ g of TPF/gm soil/24hours) at different growth stages

\begin{tabular}{|c|c|c|c|c|c|}
\hline $\begin{array}{l}\text { Sl. } \\
\text { No. }\end{array}$ & Treatments & $\begin{array}{l}\text { Active } \\
\text { tillering }\end{array}$ & $\begin{array}{l}\text { Panicle } \\
\text { initiation }\end{array}$ & Flowering & Maturity \\
\hline $\mathbf{T}_{1}$ & $100 \%$ RDF + FYM 10t /ha & 28.52 & 45.39 & 18.29 & 15.28 \\
\hline $\mathbf{T 2}$ & $\begin{array}{l}\text { 100\% RDF + FYM 5t/ha } \\
\text { +Green Manuring + } \\
\text { Biofertilizer }\end{array}$ & 29.21 & 44.12 & 20.32 & 16.34 \\
\hline$\overline{\mathbf{T}_{3}}$ & $\begin{array}{l}75 \% \text { RDF with Nimin }+ \\
\text { FYM 10t /ha }\end{array}$ & 28.81 & 38.01 & 21.98 & 15.09 \\
\hline $\mathbf{T}_{4}$ & $\begin{array}{l}75 \% \text { RDF with Nimin + } \\
\text { FYM 5t/ha + Green } \\
\text { Manuring + Biofertilizer }\end{array}$ & 26.17 & 32.23 & 17.32 & 14.97 \\
\hline $\mathbf{T}_{5}$ & STBR + FYM 10t /ha & 28.96 & 37.35 & 17.18 & 13.09 \\
\hline $\mathrm{T}_{6}$ & $\begin{array}{l}\text { STBR+ FYM 5t /ha + Green } \\
\text { Manuring + Biofertilizer }\end{array}$ & 29.23 & 64.28 & 21.30 & 16.37 \\
\hline$\overline{T_{7}}$ & $100 \% \mathrm{RDF}$ & 26.18 & 31.12 & 19.30 & 17.18 \\
\hline$\overline{T_{8}}$ & $\begin{array}{l}100 \% \text { Organic (Green } \\
\text { manuring + FYM + Mataka } \\
\text { khata+ Biofertilizer) }\end{array}$ & 28.80 & 30.03 & 16.34 & 12.03 \\
\hline
\end{tabular}

Table.8 Effect of different manures on urease activity ( $\mathrm{mg} \mathrm{NH}_{4}{ }^{+} / \mathrm{kg}$ soil/24 hours) at different growth stages

\begin{tabular}{|c|c|c|c|c|c|}
\hline $\begin{array}{l}\text { Sl. } \\
\text { No. }\end{array}$ & Treatments & $\begin{array}{l}\text { Active } \\
\text { tillering }\end{array}$ & $\begin{array}{l}\text { Panicle } \\
\text { initiation }\end{array}$ & Flowering & Maturity \\
\hline $\mathbf{T}_{1}$ & $100 \%$ RDF + FYM 10t /ha & 71.680 & 81.23 & 34.12 & 24.33 \\
\hline $\mathbf{T 2}$ & $\begin{array}{l}\text { 100\% RDF + FYM 5t/ha } \\
\text { +Green Manuring + } \\
\text { Biofertilizer }\end{array}$ & 124.887 & 132.11 & 54.12 & 32.33 \\
\hline $\mathbf{T}_{3}$ & $\begin{array}{l}75 \% \text { RDF with Nimin }+ \\
\text { FYM 10t / ha }\end{array}$ & 73.823 & 88.2 & 43.03 & 27.12 \\
\hline $\mathrm{T}_{4}$ & $\begin{array}{l}75 \% \text { RDF with Nimin + } \\
\text { FYM 5t/ha + Green } \\
\text { Manuring + Biofertilizer }\end{array}$ & 120.237 & 132.14 & 64.30 & 53.24 \\
\hline $\mathrm{T}_{\mathbf{5}}$ & STBR + FYM 10t /ha & 95.797 & 103.34 & 54.23 & 27.12 \\
\hline$\overline{T_{6}}$ & $\begin{array}{l}\text { STBR+ FYM 5t /ha + Green } \\
\text { Manuring + Biofertilizer }\end{array}$ & 132.340 & 144.2 & 73.45 & 64.23 \\
\hline $\mathrm{T}_{7}$ & $100 \% \mathrm{RDF}$ & 98.683 & 111.1 & 64.14 & 42.09 \\
\hline $\mathbf{T}_{8}$ & $\begin{array}{l}\text { 100\% Organic (Green } \\
\text { manuring + FYM + Mataka } \\
\text { khata+ Biofertilizer) }\end{array}$ & 114.327 & 124.2 & 85.23 & 73.10 \\
\hline
\end{tabular}


Gyathry found that the numbers of all aerobic bacteria in the SRI rhizosphere were increased by more than $50 \%$ before and during panicle initiation, compared to those in the rhizosphere of conventionally grown rice of same variety. The populations of Azospirillum also increased similarly, while Azotobacter, another diazotroph (N-fixing bacterium) and phosphate-solubilizing bacteria increased by even more, by about $75 \%$. During panicle initiation, the numbers of diazotrophs were more than twice as high under SRI management as with conventional practice. Throughout the crop cycle, not only were more bacteria found in SRI rhizospheres overall, but there were even more of those species that enhance plants' nutrient availability. The levels of enzymes that reflect the processes of $\mathrm{N}$ and $\mathrm{P}$ mobilization and uptake in the soil were also measured.

This showed enzyme levels significantly greater at almost all phases of crop growth when SRI practice altered the management of plants, soil, water and nutrients.

Table 4 showed that the bacteria population was increased during panicle initiation and then decreases and the same trend was followed in both population of fungi and actinomycetes. In all the treatments showed same trend. The treatment treated with STBR+ FYM 5t /ha + Green Manuring + Biofertilizer showed best result in population of bacteria, fungi and actinomycetes (Table 5 and 6).

Microorganisms produce a variety of enzymes in soil. The types of enzymes produced vary with the environment conditions. Some bacteria or fungi produce substrate-specific enzyme like cellulose, phenolase, esterase etc. only when a particular substrate is present. Some enzymes such as dehydrogenase, urease etc. are always present in cells and are produced without the influence of any substrate. Soil enzyme activities and microbial number have good correlation.

The capacity of SRI practices to affect soil bacterial populations, sometimes quite significantly, is evident from these data. The enzymatic 'footprint' of dehydrogenase is a strong indicator of biological activity within the rhizosphere, consistent with population estimates. We note that these evaluations did not assess changes in populations of mycorrhizal fungi, symbionts known to make important contributions to crop health and productivity by improving plant roots' access to both nutrients and water (Schreiner et al., 1997).

The results showed that the enzyme activity i.e., dehydrogenase and urease was more during panicle initiation stage in all the treatments showed same trend and the decreases at maturity (Table 7 and 8). The treatment treated with STBR+ FYM 5t /ha + Green Manuring + Biofertilizer showed best result in enzyme activity.

The present investigation has concluded that incorporation of soil test based fertilizer recommendation along with $5 \mathrm{tFYM} / \mathrm{ha}$, green manure and biofertilizer had showed best result in nutrient availability, microbial population and enzyme activity and also increased during panicle initiation of growth stage in all treatments under SRI cultivation of rice in both kharif and rabi season. The treatment (T2) treated with 100\%RD along with 5tFYM/ha, green manure and biofertilizer also showed on par results as T6 in nutrient availability microbial population and enzyme activity under SRI cultivation of rice.

\section{References}

Ceesay M, Reid WS, Fernandes ECM, Uphoff $\mathrm{N}$ (2007) Effects of repeated soil 
wetting and drying on lowland rice yield with system of rice intensification (SRI) methods. Intl J Agric Sust 4:5-14 Gyathry G (2002) Studies on dynamics of soil microbes in rice rhizosphere with water saving irrigation and in situ weed incorporation. Unpublished thesis, Tamil Nadu Agricultural University, Coimbatore, Tamil Nadu, India

Kabir H, Uphoff N (2007) Results of disseminating the system of rice intensification with Farmer Field School methods in Northern Myanmar. Exper Agric 43:463-476

Mishra A, Salokhe VM (2008) Seedling characteristics and early growth of transplanted rice under different water regimes. Exper Agric 44:1-19
Namara R, Bossio D, Weligamage P, Herath I (2008) The practice and effects of the system of rice intensification (SRI) in Sri Lanka. Qtly J Intl Agric 47:5-23

Thakur AK, Uphoff N, Antony E (2010) An assessment of physiological effects of system of rice intensification (SRI) practices compared to recommended rice cultivated practices in India. Exper Agric 46:77-98

Uphoff N, Iswandi A, Rupela OP, Thakur AK, Thiyagarajan TM (2009) Learning about positive plant-microbial interactions from the system of rice intensification (SRI). Asp Appl Biol 98:29-54

\section{How to cite this article:}

Sowmya Pogula and Rout, K.K. 2018. Effect of Different Manurial Practices on Nutrient Availability at Different Growth Stages under SRI Method of Rice Planting. Int.J.Curr.Microbiol.App.Sci. 7(05): 2800-2807. doi: https://doi.org/10.20546/ijcmas.2018.705.326 